\title{
The Minimum Stellar Mass in Early Galaxies
}

\author{
E. O. Vasiliev $^{1,2}$, Yu. A. Shchekinov ${ }^{1 *}$ \\ ${ }^{1}$ Rostov State University, Rostov-on-Don, Russia \\ ${ }^{2}$ Physics Research Institute, Rostov State University, Rostov-on-Don, Russia
}

\begin{abstract}
The conditions for the fragmentation of the baryonic component during merging of dark matter halos in the early Universe are studied. We assume that the baryonic component undergoes a shock compression. The characteristic masses of protostellar molecular clouds and the minimum masses of protostars formed in these clouds decrease with increasing halo mass. This may indicate that the initial stellar mass function in more massive galaxies was shifted towards lower masses during the initial stages of their formation. This would result in an increase of the number of stars per unit halo mass, i.e., the efficiency of star formation.
\end{abstract}

\section{INTRODUCTION}

In the hierarchical scenario of structure formation in the Universe, gravitationally bound objects - dark matter halos, in which stars can form - first appear at redshifts $z \sim 20$ and have masses $M \sim 10^{6} M_{\odot}$, including the dark and baryonic components [1-3]. These halos form due to multiple collisions and merging of similar objects of smaller mass. The dynamics of the early stages of these processes are determined by the gravitational instability of the dark component, which consists of cool (non-relativistic) particles. Density perturbations of practically any wavelength can develop in the dark component, since the minimum critical scale of the perturbations - the Jeans mass, which depends on the velocity dispersion in a collisionless gas - is negligible compared with the mass of the halo (see, for example, [4]). The spatial scale of the perturbations can be constrained from below by a truncation of the perturbation spectrum. The amplitude of the density perturbations on small scales is known to decrease with wavelength as a power law $\propto \lambda^{-3}[5,6]$. Thus, the formation of structure includes relatively developed shortwavelength motions modulated by slowly growing long-wavelength flows [7].

*yus@phys.rsu.ru 
Under these conditions, a nearly one-dimensional motion develops, in which perturbations are compressed mainly along a single direction, giving rise to a disk-like configuration $[8,9]$. Baryons confined by the gravitation field of the dark matter and involved in this one-dimensional flow undergo shock compression and are heated to the temperature $T_{f} \sim m_{p} v_{c}^{2} / 2 k$, where $v_{c}$ is the velocity of the inward gas flow; as a result, a dense layer or disk is formed. The compressed baryonic layer is efficiently cooled by radiative processes by neutral hydrogen atoms or molecules. The formation of molecular hydrogen is substantially enhanced behind shock fronts, so that the cooling of the gas may basically be governed by the $\mathrm{H}_{2}$ molecules. When the temperature decreases to $200 \mathrm{~K}$, deuterium efficiently transforms into HD, which results in an even higher thermal energy loss rate, providing favorable conditions for the fragmentation of the compressed gas [10-17]. Thus, during the formation of galaxies, converging flows result in an emergence of dense baryonic condensations in which stellar objects can subsequently form. This concept was recently discussed in $[16,17]$. During the formation of massive protogalaxies, dense baryonic layers are maintained over longer times, so that the gas is cooled substantially and higher densities are reached. Therefore, one may expect that during the formation of more massive galaxies, baryonic fragments of lower mass can become gravitationally unstable, and less massive stars can be formed, which increases the number of stars per unit mass of the halo - in other words, the star formation efficiency. Here, we study this possibility.

Section 2 discusses the model adopted to describe the thermal evolution of baryons behind shock fronts. Section 3 presents the results, and Section 4 contains our conclusions and final discussion. Our calculations were based on the $\Lambda \mathrm{CDM}$ model for the Universe: $\left(\Omega_{0}, \Omega_{\Lambda}, \Omega_{m}, \Omega_{b}, h\right)=(1.0,0.71,0.29,0.047,0.72)$, with the assumed deuterium abundance $n[D] / n=2.6 \cdot 10^{-5}[18]$.

\section{HALO VIRIALIZATION AND THE THERMO-CHEMICAL EVOLUTION OF GAS}

In the linear stage of its growth, the evolution of a dark-matter density perturbation $\delta_{d m}(t)$ can be described by the equation (see e.g. [6]):

$$
\ddot{\delta}_{d m}+2 H \dot{\delta}_{d m}=\frac{3}{2} H^{2}\left(\Omega_{b} \delta_{b}+\Omega_{d m} \delta_{d m}\right),
$$

where $H$ is the Hubble constant, $\delta_{b}$ is the perturbation of the baryon density, and $\Omega_{d m}=\Omega_{m}-\Omega_{b}$. As seen from this equation, there is no limiting minimum scale in dark matter. In reality, the Jeans mass in collisionless dark matter depends on the velocity dispersion; however, this value 
turns out to be negligibly small compared with the mass of the halo. As was already noted, if a density perturbation in dark matter is considerably overcritical, then its compression will proceed more rapidly along one direction $[8,9]$. The dissipative baryonic component follows the dark matter potential, and forms a disk-like configuration in the symmetry plane. The growth of the perturbation is essentially a collision between flows of dark matter and baryons, and results in the formation of a shock front in the baryonic component. Subsequently, the collisionless dark matter becomes virialized via violent relaxation [19], and the baryonic component undergoes shock compression in the colliding flows, forming a dense radiatively cooling layer. Starting from some time, the increased density of the gas may exceed the density of the dark matter, and the dynamics of the gas will be determined by its own parameters. Therefore, we can neglect the influence of the dark matter and consider only the baryonic component. This is valid at least within the characteristic collision time between regions, $t_{d} \sim D / v$.

When gas flows collide, a thin dense gas layer and two diverging shock fronts around the contact region are initially formed; behind the shock fronts, the gas is heated to the temperature $T_{f} \sim m_{p} v_{c}^{2} / 2 k$, where $m_{p}$ is the proton mass and $v_{c}$ the collisional velocity. The compression and formation of the dense layer lasts approximately $t \sim t_{d}=D / v_{c}$. In the transverse direction, the gas is not restrained by dynamical pressure and can expand freely on a time scale $t_{\perp} \sim D / c_{s}$, where $c_{s}$ is the sound speed. Thus, the ratio of the characteristic time scales is $t_{d} / t_{\perp} \simeq 1 / \mathcal{M}$, where $\mathcal{M}$ is the Mach number, and we have for a collision between baryonic flows with velocities $v_{c}>c_{s}$ the condition $t_{d}<t_{\perp}$. Therefore, for supersonic collisions, we can neglect the transverse motion in the layer when $t<t_{d}$ and solve a one-dimensional problem. It was shown via twodimensional modeling that, indeed, only an insignificant amount of the mass is lost to transverse outflow during a collision of gas clouds [20]. The velocity of the gas motions during the formation of a dark halo with mass $M$ is

$$
v_{c}=\sqrt{3} \sigma
$$

where

$$
\sigma(M)=\sqrt{G M^{2 / 3}\left(3 \pi^{3} \Omega_{m} \rho_{0}\right)^{1 / 3}(1+z)}
$$

$\sigma$ is the one-dimensional velocity dispersion and $\rho_{0}$ is the present mean total density of the Universe. It is clear that the collisional velocity and gas temperature behind the shock fronts will be higher when more massive halos are formed.

The thermal evolution of baryons behind a shock front can be described by a system of ordinary differential equations written for a single Lagrangian element of the fluid:

$$
\dot{x}=\beta x n(1-x-2 f)-k_{1} n x^{2},
$$




$$
\begin{gathered}
\dot{f}=k_{m} n(1-x-2 f) x, \\
\dot{g}=k_{D 1} f x n d_{c}-n x\left(k_{D 1} f+k_{D 2}\right) g, \\
\dot{T}=\frac{2}{3} \frac{\dot{n}}{n} T+\Sigma \Lambda_{i} .
\end{gathered}
$$

where $x=n(\mathrm{e}) / \mathrm{n}, f=n\left(\mathrm{H}_{2}\right) / \mathrm{n}, g=n(\mathrm{HD}) / \mathrm{n}$ are the relative concentrations of electrons, $\mathrm{H}_{2}$, and $\mathrm{HD}, d_{c}=n(\mathrm{D}) / \mathrm{n}$ is the cosmological abundance of deuterium, $k_{i}$ are the rates of reactions [3, 21], $\beta$ is the rate of collisional ionization of hydrogen [14], $\Lambda_{i}$ is the rate of cooling and heating due to Compton interactions with photons of the cosmic microwave background radiation (CMB), emission in atomic and molecular hydrogen lines [22], and in HD lines [21, 23, 24]. Initially, the gas density behind the shock front is $n=4 n_{0}$, where $n_{0}$ is the density before the shock front. Further, we will assume that each element of the gas behind the shock front evolves isobarically, and that the density is described by the equation

$$
n=\frac{p}{\mu k T}
$$

where $\mu=\rho / n m_{p}$.

In the $\Lambda \mathrm{CDM}$ scenario, the spectrum of perturbations in the dark matter is rather steep $(n=-3)$, which implies that halos with masses of $10^{4}-10^{8} M_{\odot}$ condense out over times shorter than the corresponding Hubble time [25]. A certain time after forming, the halos become virialized. In addition, since they are involved in large-scale motions, they merge with each other, forming halos of higher mass. Both the virialization and the merging occur on comparable times. For this reason, two limiting possibilities can be suggested for the initial conditions for the halo formation. In the first, halos of smaller mass (subhalos) collide and soon reach the virial state at larger redshifts. In the second, virialization and merging of subhalos occur at the same time at a given redshift. In both cases, the process can be presented as a one-dimensional compression. The two cases differ in the initial characteristics of the matter in the flow; more precisely, in the density and the velocity of the collision. In the first model, a halo is formed due to collisions of subhalos, and the parameters of the matter correspond to those in the objects that have been virialized by the beginning of the formation of the larger halo $\left(z_{t a}\right)$, i.e., by the time it separates from the cosmological expansion. The matter density can be taken to be $\rho \simeq 18 \pi^{2} \rho_{0}\left(z_{t a}\right)$ [25], and the relative concentrations of e, $\mathrm{H}_{2}$, and $\mathrm{HD}$ to their values inside the subhalos. In the second model, subhalos are virialized and collide at the same redshift, and the initial density of the matter is $\rho \simeq 18 \pi^{2} \rho_{0}\left(z_{v i r}\right)$, where $\rho_{0}(z)$ is the background density of matter at the redshift $z$; the relative concentrations of electrons, $\mathrm{H}_{2}$, and $\mathrm{HD}$ are assumed to be equal to their values inside the halo. In both models, the collision results in the formation of shock fronts, behind 
which gas is rapidly heated to the temperature $T_{f}=m_{p} v_{c}^{2} / 3 k$, which is taken as the initial temperature of an element of gas behind the front. Since the initial conditions in the models considered differ only in their densities, the results will be qualitatively similar. Therefore, we will describe only the first model, but present graphs for both. Calculations for halos with the masses corresponding to $3 \sigma$ perturbations were carried out only for the second model.

\section{RESULTS}

\subsection{Thermal and Chemical Evolution of Baryons}

Let us consider the chemical and thermal evolution of the gas behind the shock front using the example of halos formed at the redshift $z=15$. Figure 1 presents the concentrations of electrons, $\mathrm{H}_{2}$, and $\mathrm{HD}$ and the temperatures reached in the gas behind the shock front in the end of one compression time during the formation of a halo with mass $M$. It is clearly seen that, starting from a certain value of halo mass, the relative concentration of $\mathrm{H}_{2}$ molecules exceeds $5 \cdot 10^{-4}$ and the temperature of the gas decreases substantially $(T \sim 500 \mathrm{~K})$. This corresponds to the minimum $\mathrm{H}_{2}$ concentration needed in order for the gas to cool rapidly, in one comoving Hubble time [3]. In more massive halos, the $\mathrm{H}_{2}$ concentration continues to grow, and can reach $10^{-2}$ for halos with $M_{h} \gtrsim 4 \cdot 10^{7} M_{\odot}$. Radiative losses in $\mathrm{H}_{2}$ lines cool the gas to $T \lesssim 200 \mathrm{~K}$, and all the deuterium becomes rapidly bound in HD molecules due to the effects of chemical fractionation. Due to strong cooling in HD lines, the temperature drops to several tens of Kelvin, which is close to the temperature of the CMB radiation at this redshift $2.73(1+z)$. HD molecules provide efficient heat exchange between the CMB and baryons, due to the fact that they absorb background photons and subsequently transfer the excitation energy to the gas through collisions $[24,26,27]$. Under these conditions, the Jeans mass behind the shock front (dashed curves in lower panels of Fig. 1) decreases and, starting from some value, becomes substantially lower than the baryonic mass of the forming halo $\left(\Omega_{b} M_{h} / \Omega_{m}\right.$, solid curve). For example, for a halo with $M=10^{7} M_{\odot}$, the Jeans mass is only $M_{J} \simeq 10^{3} M_{\odot}$.

Let us now consider the instability of the cool compressed layer and possible formation of dense baryonic condensations in it. We will assume that the gas in the layer is gravitationally unstable and can fragment provided the critical perturbation length $\lambda_{m}$ is shorter than the initial size of the cloud $D$, and the corresponding time $t_{m}$ is shorter than the compression time [28, 29]:

$$
\lambda_{m} / D \leq 1, \quad t_{m} / t_{d} \leq 1
$$


The vertical line in Fig. 1 indicates the minimum halo mass for which the criterion (9) is fulfilled during its formation. Thus, the gas behind the shock front originating during the formation of a halo with mass $M \geq 10^{7} M_{\odot}$ at a redshift $z \simeq 15$ is unstable by the end of the compression phase; accordingly, the first baryonic objects with masses roughly equal to the Jeans mass $M_{J} \leq 10^{3} M_{\odot}$ can form inside the halo. Since the temperature of the gas in the unstable fragments is lower than $200 \mathrm{~K}$, the abundance of HD also increases substantially, thereby determining the subsequent thermal evolution of the gas behind the shock fronts forming during the formation of the first halos.

Figure 2 shows the interrelation between the redshift and the minimum halo mass for which the layer behind the shock front becomes during its formationunstable according to the criterion (92). For comparison, the mass of $3 \sigma$ perturbations is displayed, as well as the minimum mass for which baryons in already virialized halos can cool and form gravitationally bound objects [3]. For instance, a gravitationally unstable gas layer is formed during the virialization behind the shock front in a halo with mass $M_{\text {min }} \simeq 5 \cdot 10^{6} M_{\odot}$ at $z=20$. Note that this derived minimum halo mass is comparable to that obtained in [3], however, the temperature of the gas is substantially lower in our model.

Baryonic objects with masses approximately equal to the Jeans mass can form inside this unstable layer. Figure 3 displays the dependence of the Jeans mass behind the shock front on the time at which the halo forms; the dashed line corresponds to the minimum halo mass needed in order for the layer behind the shock front to be unstable according to (9), and the solid curve to the mass of $3 \sigma$ perturbations. It is seen that at the given redshift, the Jeans mass in the layer formed behind the shock front decreases with increasing halo mass: the conditions are favorable for forming fragments with lower masses in more massive halos. For example, in the formation of a halo with mass $10^{7} M_{\odot}$ (which corresponds to the minimum halo mass) at redshift $z=15$, the layer behind the shock front becomes unstable, and the baryonic objects formed there can have masses of $M_{b} \sim 3 \cdot 10^{3} M_{\odot}$, while $M_{b} \sim 300 M_{\odot}$ for a halo with mass $10^{8} M_{\odot}$. Fragments with masses as low as $M_{b} \sim 100 M_{\odot}$ will be unstable in a halo with mass $3 \cdot 10^{9} M_{\odot}$ formed at $z=10$. The possible masses of gravitationally unstable baryonic condensations lie within the above limits which widen with decreasing $z$.

During the formation of a massive halo, the gas behind the shock front cools and is compressed more intensely, due to the more efficient formation of $\mathrm{H}_{2}$ molecules behind more strong shock fronts. Figure 4 presents the dependence of the gas density on the mass of the halo and the redshift at which it begins to form. The variation of the slope of the $n(M)$ curves with increasing 
$M$ reflects the fact that, by the end of the compression phase, the temperature behind the shock front reaches the temperature of the $\mathrm{CMB}$, due to the efficient formation of $\mathrm{HD}$ molecules and energy exchange between the gas and CMB radiation via the absorption of background photons by HD molecules followed by collisional deexcitation. The final density at a given $z$ depends only on the initial temperature of the gas behind the shock front, which, in turn, is a function of the halo mass: $n=n_{0}\left(T_{f} / T_{C M B}\right)$.

\subsection{The Minimum Mass of Baryonic Objects}

Baryonic fragments with masses $10^{2}-10^{4} M_{\odot}$ (Fig. 3) formed behind the shock front in an unstable layer are optically transparent to radiation in $\mathrm{H}_{2}$ and $\mathrm{HD}$ lines. Compression of the fragments occurs in an isothermal regime at temperature close to $2.73(1+z) \mathrm{K}$, as is mainly ensured by the HD molecules.

In the isothermal regime, a characteristic self-similar density profile is formed, $\rho \sim r^{-2}[30$, 31], the optical depth in the HD lines increases in the central regions, and the Jeans mass gradually decreases. The critical point is the formation of an optically thick $(\tau \geq 1)$ core region with a mass equal to or exceeding the Jeans mass. In the central regions when $\tau \sim 1$, the gas density is high, $\left(\sim 10^{9}-10^{10} \mathrm{~cm}^{-3}\right)$, and all the hydrogen is transformed into the molecular phase via three-body reactions. However, due to the low temperature of the gas, the contribution of $\mathrm{H}_{2}$ molecules to the cooling in optically transparent regions - and, therefore, their impact on the dynamics of the compression - is unimportant. The formation of an opaque core is fully determined by the HD molecules in the gas. After the formation of an opaque, gravitationally unstable core, the compression regime changes from isothermal to adiabatic. Figure 5 presents the dependences of the density, optical depth, temperature, and Jeans mass inside the cloud when this state has been reached by $z \simeq 15.3$ for a fragment with mass $M=500 M_{\odot}$ formed as a result of instability in the layer during the formation of a halo with $M_{h}=2 \cdot 10^{7} M_{\odot}$ at $z_{v}=17$. It is clear that the temperature through the cloud is almost constant and close to that of the CMB. The formed opaque core has a density of $\geq 10^{10} \mathrm{~cm}^{-3}$ and a mass of $\sim 0.15 M_{\odot}$.

Figure 6 presents the dependence of the mass of the opaque core on the mass of the halo for the limiting masses: the minimum mass $M_{\text {min }}$ needed for the layer behind the shock fronts to be unstable according to (9) for both models of the initial conditions, and the mass corresponding to $3 \sigma$ perturbations, $M_{3 \sigma}$. The numbers in Fig. 6 denote the redshift at which the halo is virialized. It is seen that the mass of the opaque core decreases as the mass of the halo increases: the mass of the core for a halo mass of $M_{h} \simeq 2 \cdot 10^{7} M_{\odot}$ at $z=17$ is $M(\tau \geq 1) \sim 0.15 M_{\odot}$, while the 
mass of the core is only $M(\tau \geq 1) \sim 0.06 M_{\odot}$ for a halo mass $M_{h} \simeq 10^{9} M_{\odot}$ at $z=12$.

Thus, the formation of dark halos results in the formation of strong shock fronts in the baryonic component, substantial cooling, and an increase in the gas density. The cold postshock gas layer is unstable against formation of baryonic condensations in which protostellar cores can in turn be formed. The higher the mass of the initial halo, the lower the characteristic mass of the gravitationally unstable fragment - a possible protostellar cluster or an opaque protostellar core.

\subsection{Thermal Instability}

The development of thermal instability becomes possible in a radiatively cooling gas under certain cooling regimes [32]. In a non-steady-state case, when radiative losses are not balanced by heating, the condition for this is

$$
\frac{d \ln \Lambda}{d \ln T}<2,
$$

where $\Lambda$ is the effective radiative-loss function (see, e.g., [33]). Figure 7 shows the temperature dependence of the function $d \ln \Lambda / d \ln T$ in the whole range of temperatures encompassed by the gas cooling behind the shock front. It is obvious that thermal instability can develop always whenever the cooling is determined by $\mathrm{H}_{2}$ and $\mathrm{HD}$ molecules, i.e., when $70 \mathrm{~K}<T<8000 \mathrm{~K}$. To order of magnitude, the characteristic size of the region of instability coincides with the size of the region behind the shock front where the gas is cooled from $T=T_{f}$. Therefore, it is obvious that the forming through thermal instability condensations will facilitate the subsequent gravitation fragmentation of the compressed layer.

\section{DISCUSSION AND CONCLUSIONS}

Collisions of baryonic flows during the formation of the first protogalaxies are accompanied with an intense cooling of the gas, which promotes fragmentation of the gas onto condensations with characteristic masses close to the masses of the present day molecular clouds. The subsequent cooling and compression of such condensations may result star formation. The higher the mass of the galaxy, the more intense the gas cooling and the smaller the mass of the molecular cloud and the minimum mass of stars formed through subsequent fragmentation. Thus, in this picture, we expect that the initial mass function of stars and initial luminosity function of stellar clusters will be shifted towards smaller values in more massive galaxies.

The formed low-mass fragments cool rapidly, due to high abundances of $\mathrm{H}_{2}$ and $\mathrm{HD}$ molecules. Starting from certain time, a protostellar cloud converges to isothermal compression phase, since 
the gas temperature is maintained near the CMB temperature due to an efficient heat exchange between the CMB radiation and baryons, which absorb background photons and then transfer the excitation energy to the gas in collisional processes [24, 26, 27]. Further, as the density in the central regions of the fragment increases, an opaque core with a mass of $\left(10^{-1}-10^{-2}\right) M_{\odot}$ is formed. This core evolves then into a hydrostatic protostellar core with a lower mass of the order of $10^{-3} M_{\odot}[30,31]$, onto which matter gradually accretes, with an accretion rate determining the final mass of the star [34]. In this process, practically all gravitational energy of the accreted gas is radiated in $\mathrm{H}_{2}$ and $\mathrm{HD}$ lines. A possibility to observe $\mathrm{H}_{2}$ line emission was discussed in [35-38]; it was found, however, that it is extremely difficult to detect this emission with either currently operating or planned telescopes [38]. For example, if $\sim 2000$ protostellar objects are formed behind the shock front during the virialization of a halo with mass $3 \cdot 10^{7} M_{\odot}$ (which corresponds to approximately $10 \%$ of the mass of baryons being transformed into protostellar fragments), the luminosity of this cluster in the $\mathrm{H}_{2} 2.34 \mu \mathrm{m}$ line will be $\sim 10^{38} \mathrm{erg} / \mathrm{s}$. With a spectral resolution of $R \sim 1000$, this corresponds to a flux from the object of $\sim 10^{-2} \mu \mathrm{Jy}$ at redshift $z=15$, while the sensitivity of the next planned SAFIR space telescope ${ }^{1}$ ) is $1 \mu \mathrm{Jy}$.

\section{ACKNOWLEDGMENTS}

The authors thank the referee for useful comments.

\section{References}

[1] T. Abel, P. Anninos, Y. Zhang and M.L. Norman, New Astr., 2, 181 (1997)

[2] V. Bromm, P. Coppi, R. Larson, R., Astrophys. J. 564, 23 (2002)

[3] M. Tegmark, J. Silk, M.J. Rees, et al., Astrophys. J. 474, 1 (1997)

[4] L. P.Grishchuk and Ya. B. Zel'dovich, Astron. Zh. 58, 472 (1981) [Sov. Astron. 25, 267 $(1981)]$

[5] P.J.E. Peebles, Astrophys. J. 263, L1, (1982)

[6] E. Kolb and M. Turner, The Early Universe (Addison-Wesley, Readwood City, 1990)

[7] P. J. E. Peebles, Principles of Physical Cosmology (Princeton Univ. Press, Princeton, 1993)

\footnotetext{
${ }^{1}$ http://safir.jpl.nasa.gov
} 
[8] C. Lin, L. Mestel, F. Shu, Astrophys. J. 142, 1431 (1965)

[9] Ya. B. Zel'dovich, Astrofiz. 6, 119 (1970)

[10] J. Smith, Astrophys. J. 238, 842 (1980)

[11] A. A. Suchkov, Yu. A. Shchekinov, and M. A. . Edel'man, Astrofiz. 18, 629 (1982) [Astrophys. 18, $360(1982)]$

[12] C. Struck-Marcel, Astrophys. J. 259, 116 (1982)

[13] C. Struck-Marcel, Astrophys. J. 259, 127 (1982)

[14] P.R. Shapiro, H. Kang, Astrophys. J. 318, 32 (1987)

[15] Yu. A. Shchekinov, Astrophys. Space Sci. 175, 57 (1991)

[16] M. Yamada, R. Nishi, Astrophys. J. 505, 148 (1998)

[17] R. Cen, Astrophys. J. 624, 485 (2005)

[18] D. N. Spergel, L. Verde, H.V. Peiris et al, Astophys. J. Suppl. Ser. 148, 175 (2003)

[19] D. Lynden-Bell, Monthly Notices Roy. Astron. Soc. 136, 101 (1967)

[20] F. Miniati, T. W. Jones, A. Ferrara and D. Ryu, Astrophys. J. 491, 216 (1997)

[21] R. Barkana, A., Loeb, Phys. Rept., 349, 125 (2001)

[22] D. Galli, and F. Palla, Astron. Astropys. 335, 403 (1998)

[23] D. Hollenbach and C.F. McKee, Astrophys. J. Suppl. Ser. 41, 555 (1979)

[24] D. Flower, Monthly Notes Roy. Soc., 318, 875 (2000)

[25] D. Puy, and M. Signore, NewA 3, 247 (1998)

[26] D. A. Varshalovich and V. K. Khersonskii, Pis'ma Astron. Zh. 2, 574 (1976) [Sov. Astron. Lett. 2, 227 (1976)]

[27] D. Galli, and F. Palla, Planet. Space Sci. 12-13, 1197 (2002)

[28] M. Stone, Astrophys. J. 159, 277 (1970)

[29] D. Gilden, Astrophys. J. 279, 335 (1984) 
[30] K. Omukai, R. Nishi, Astrophys. J. 508, 141 (1998)

[31] E. Ripamonti, F. Haardt, A. Ferrara, et al., Monthly Notices Roy. Astron. Soc. 334, 401 (2002)

[32] G.B. Field, Astrophys. J. 142, 531 (1965)

[33] Yu. A. Shchekinov, Astron. Zh. 55, 311 (1978) [Sov.Astron. 22, 182 (1978)]

[34] K. Omukai, F. Palla, Astrophys. J. 589, 677 (2003)

[35] H. Kamaya, J. Silk, Monthly Notices Roy. Astron. Soc. 332, 251 (2002)

[36] H. Kamaya, J. Silk, Monthly Notices Roy. Astron. Soc. 339, 1256 (2003)

[37] K. Omukai, T. Kitayama, Astrophys.J. 599, 738-745 (2003)

[38] H. Mizusawa, R. Nishi, and K. Omukai, Publ. Astron. Soc. Jpn. (2005) (in press); astro-ph/0404333 (2004) 


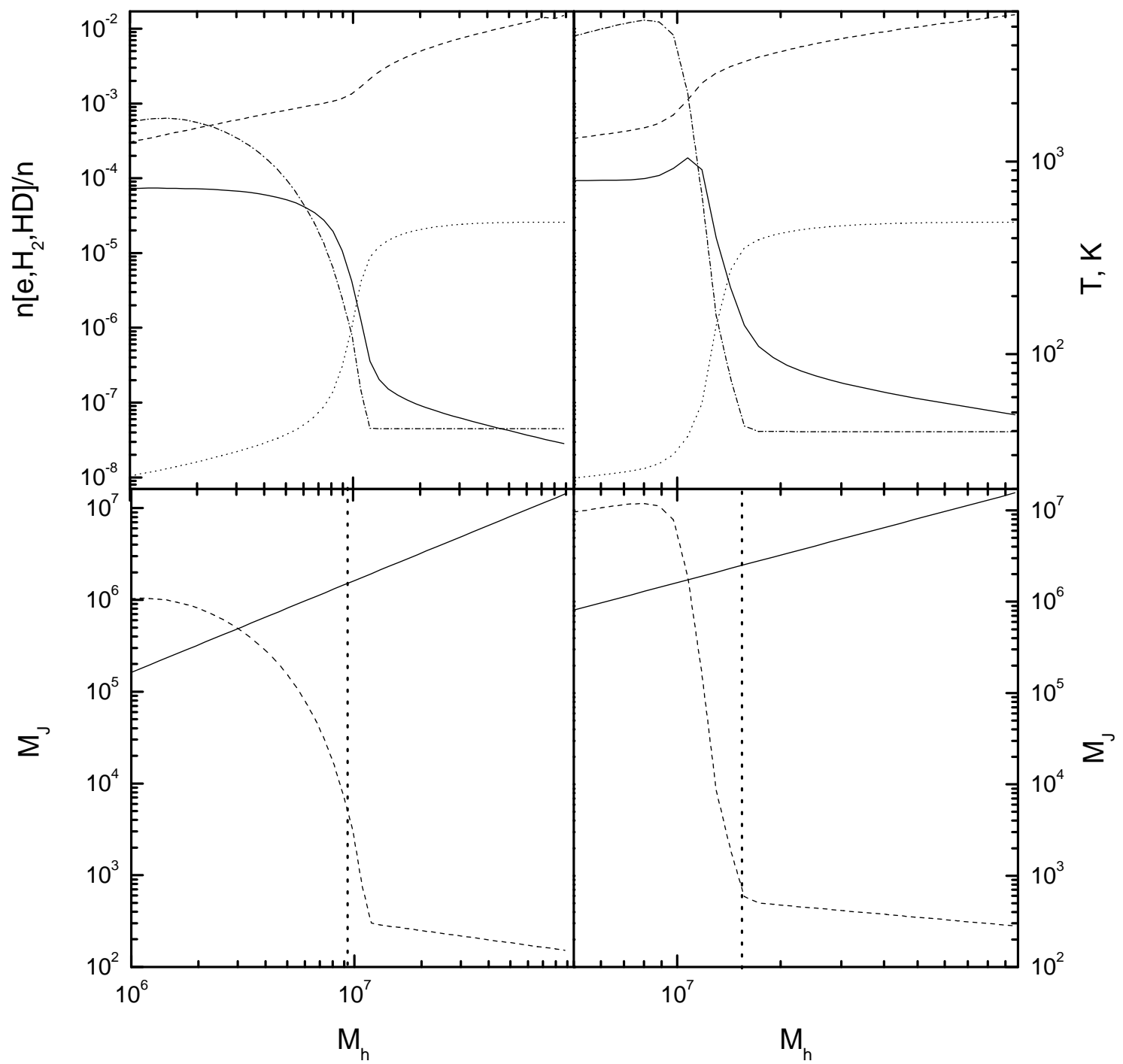

Figure 1: Top: concentrations of electrons (solid curve), $\mathrm{H}_{2}$ (dashed curve), and HD (dotted curve), and the temperature (dot-dashed curve) reached in the gas during virialization vs the halo mass $M$ at $z=15$. Bottom: total baryonic mass in the halo (solid curve) and the Jeans mass in the layer behind the shock front. The left and right panels correspond to the first and second models for the initial conditions (see the text for more details). 


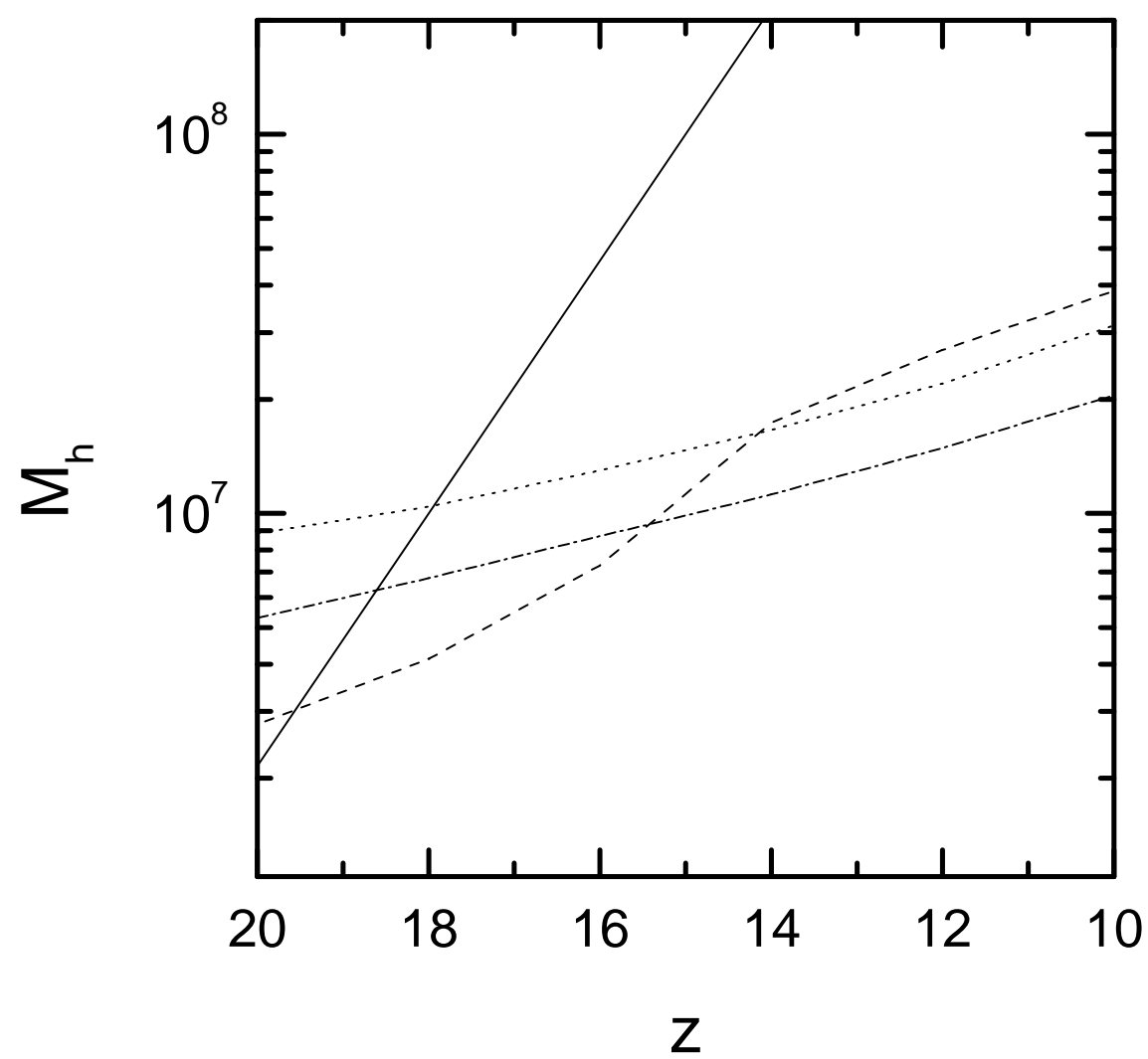

Figure 2: The minimum halo mass for which the gas layer behind the shock front becomes unstable according to the criterion (9) during the formation of the halo for two models of halo formation (dot-dashed and dotted curves). The solid line indicates the mass of $3 \sigma$ perturbations, and the dashed line the minimum mass for which baryons can cool and form gravitationally bound objects in virialized halos [3]. 


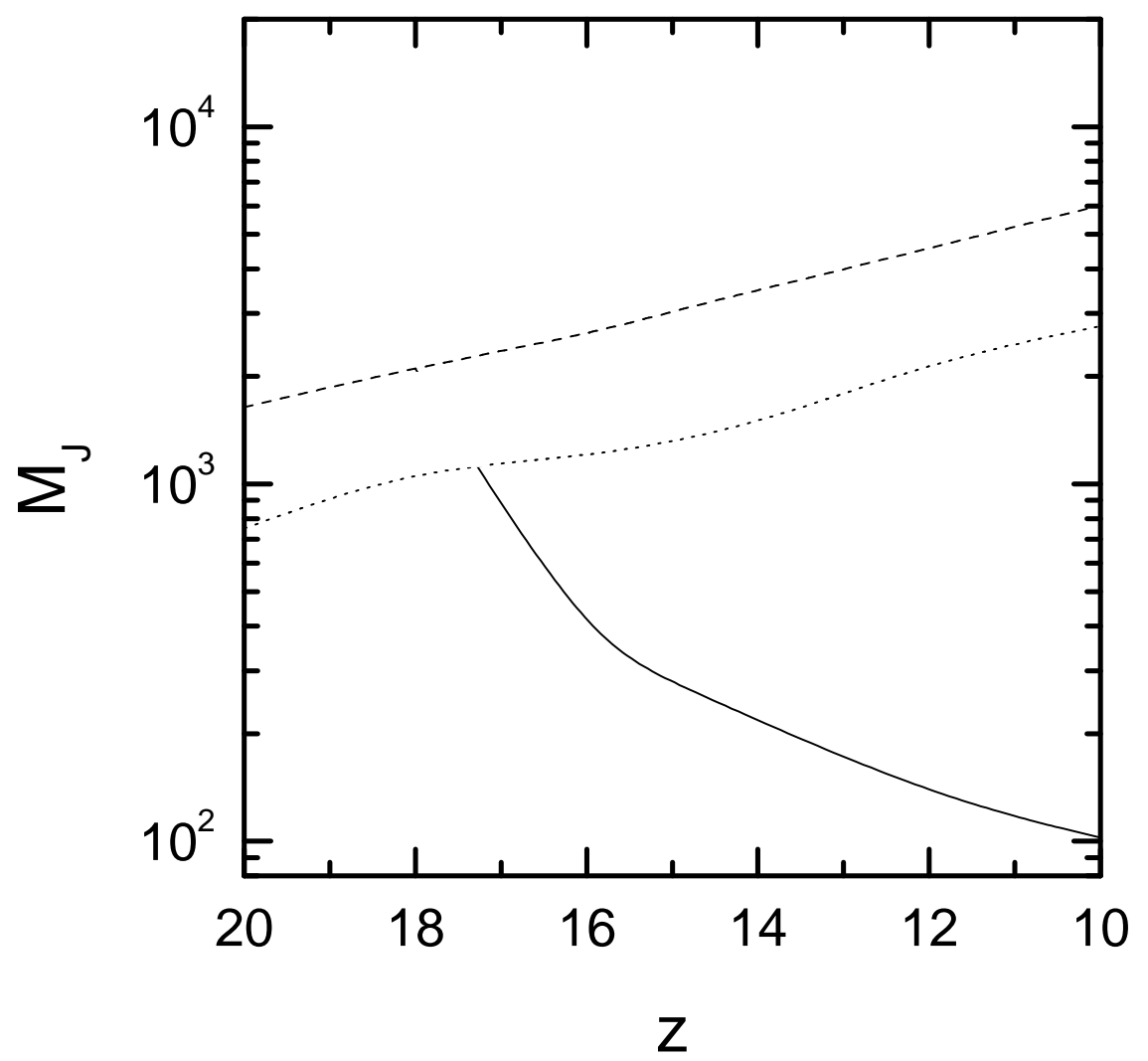

Figure 3: Dependence of the Jeans mass behind the shock front on the time when the halo forms. The dashed and dotted lines correspond to the minimum halo masses needed in order for the layer behind the shock front to be unstable according to criterion (9) for the first and second models, respectively. The solid line corresponds to the mass of the $3 \sigma$ perturbations. 


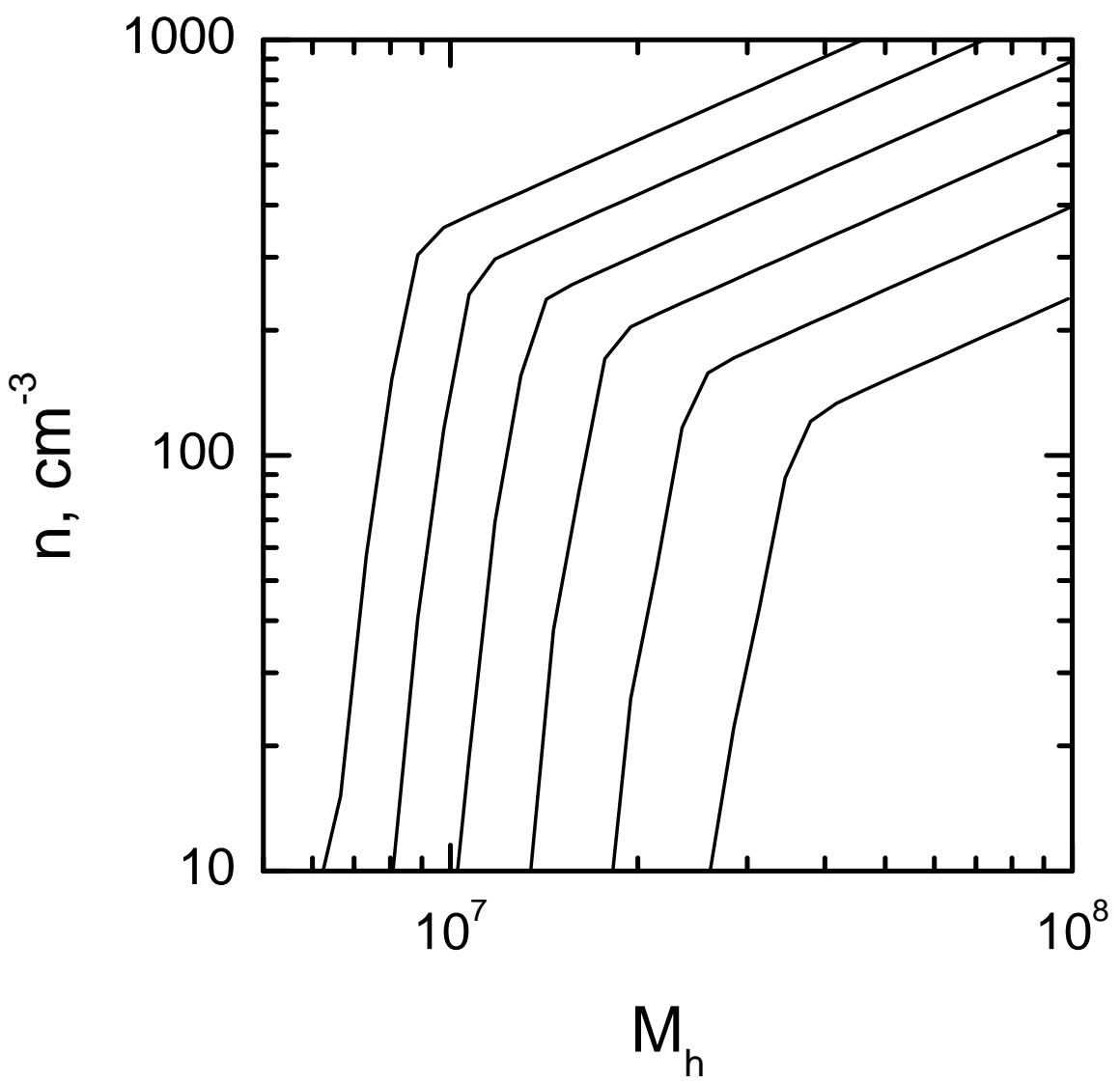

Figure 4: Dependence of the final density of the gas behind the shock fronts on the halo mass and the initial redshift at which the halo forms for $z=20,18,16,14,12,10$ (left to right). 


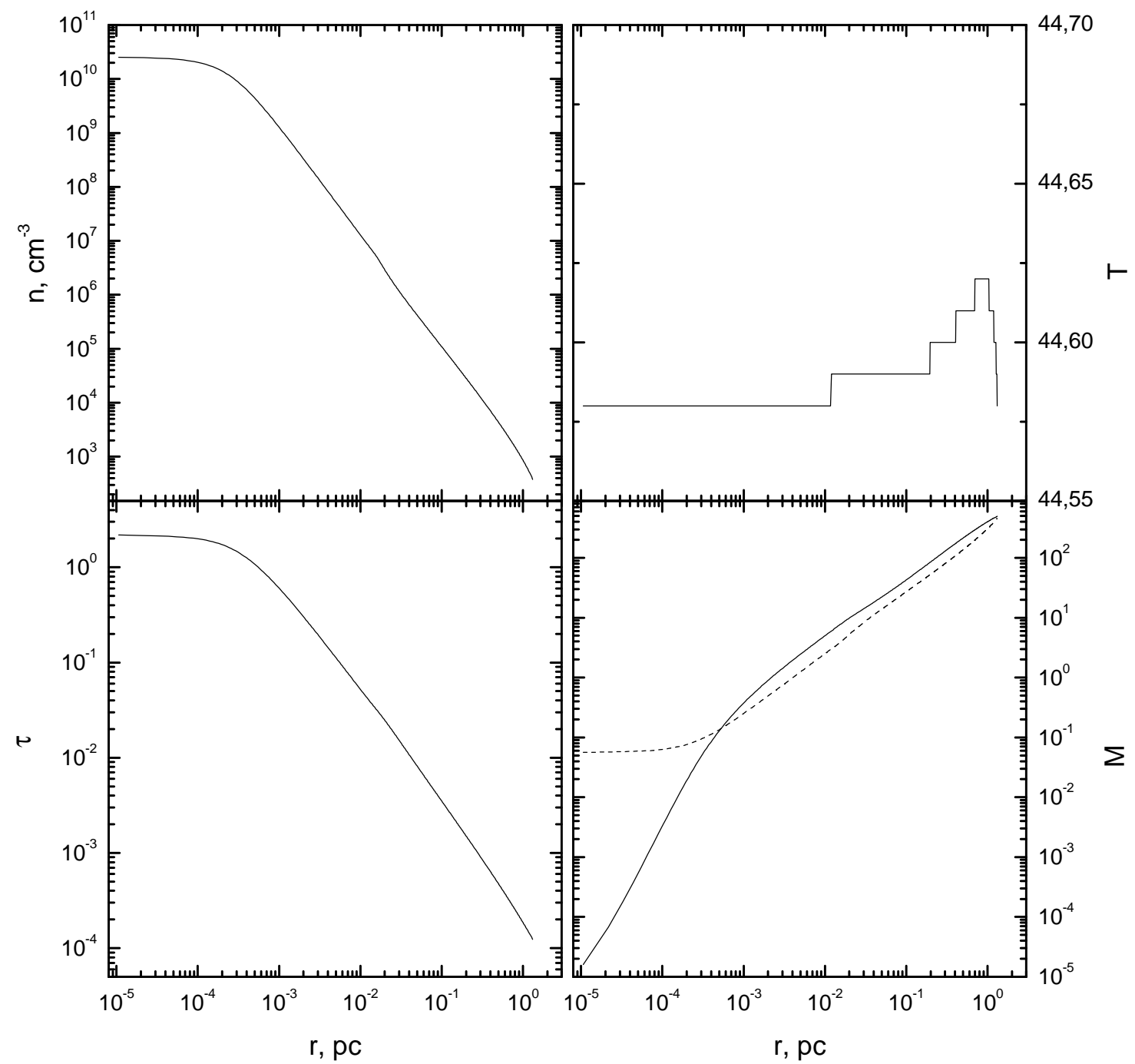

Figure 5: Density, optical depth, temperature, and Jeans mass inside a cloud at the time of formation of an opaque gravitationally unstable core $(z \simeq 15.3)$ for a fragment with mass $M=$ $500 M_{\odot}$ formed due to instability of the layer during the formation of a halo with $M_{h}=2 \cdot 10^{7} M_{\odot}$ at $z_{v}=17$. The dashed line in the lower right panel indicates Jeans mass, the solid line depicts the mass inside the corresponding radius. 


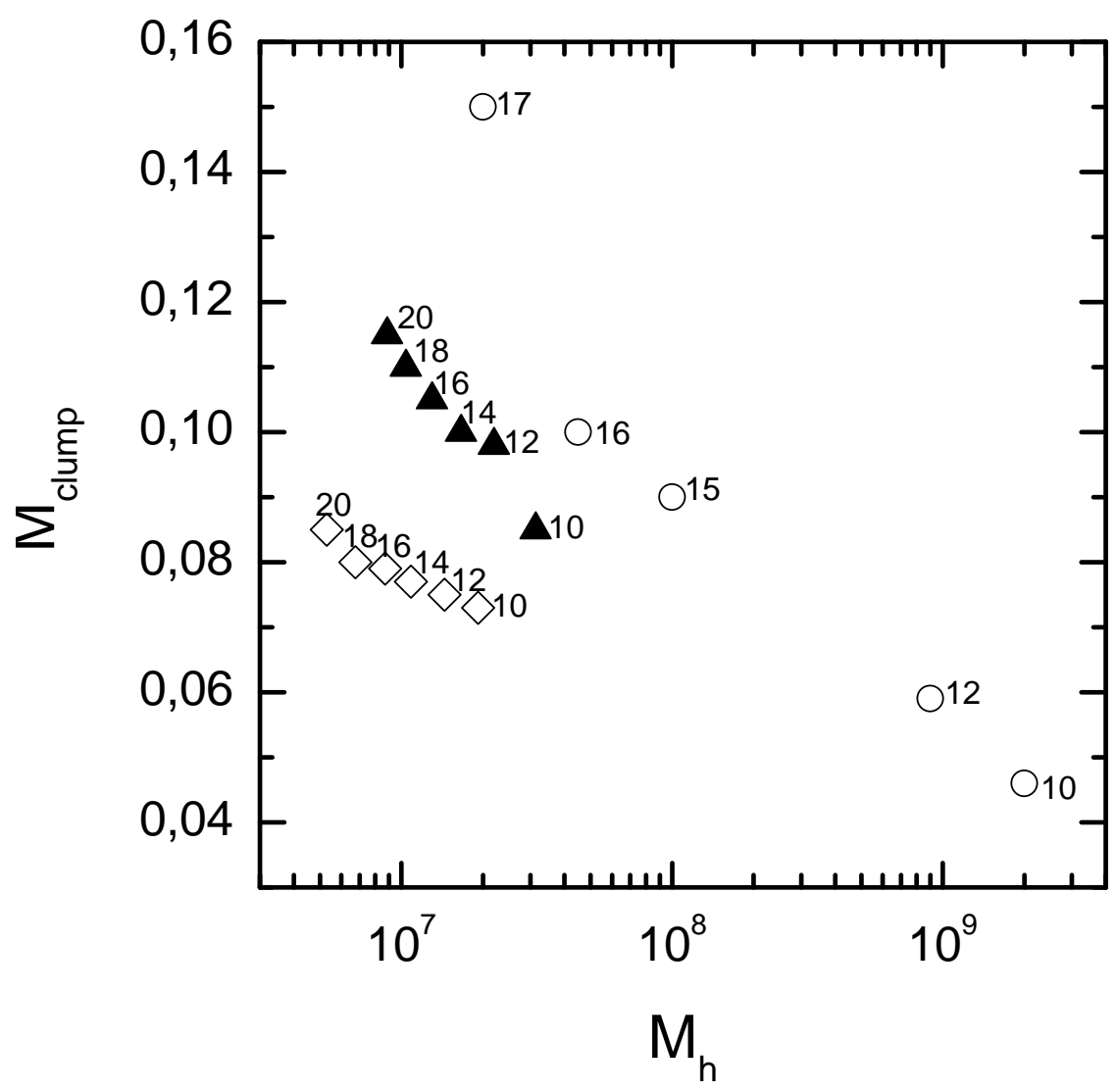

Figure 6: Dependence of the mass of the opaque core on the mass of the halo. The case of the minimum mass $M_{\text {min }}$ needed for the layer behind the shock front to be unstable according to (92) for the first and second models for the initial conditions are indicated by open diamonds and filled triangles, respectively. The case of the mass corresponding to $3 \sigma$ perturbations, $M_{3 \sigma}$, is indicated by open circles. The numbers near the symbols denote the redshift of the halo virialization. 


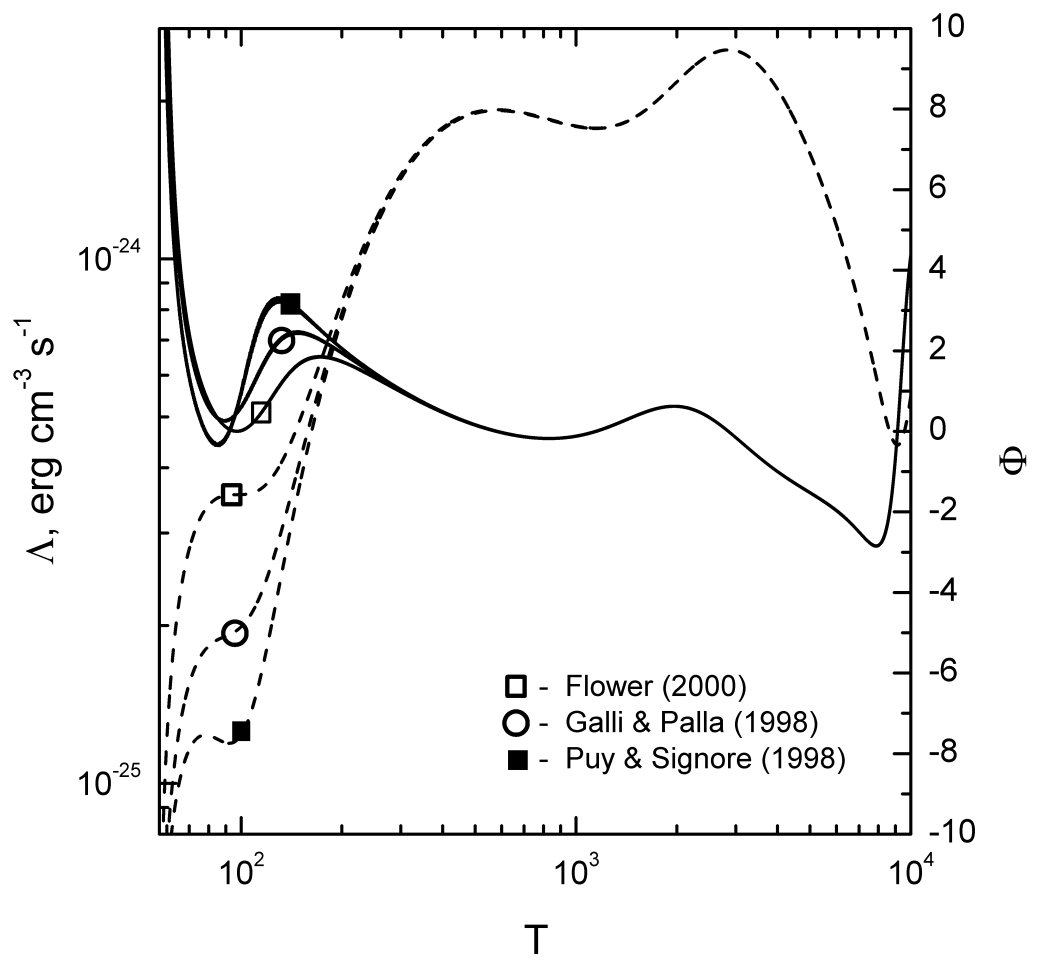

Figure 7: Temperature dependence of the cooling rate and its logarithmic derivative $\Phi=$ $d \ln \Lambda / d \ln T$ for different approximations of the radiative losses in HD lines. 